\title{
Web 2.0 tools as framework for participation and co-creation
}

Igor Bizjak [0000-0002-387 I-3 I I 6], Urban Planning Institute of the Republic of Slovenia, Ljubljana, Slovenia. Igor.bizjak@uirs.si

\begin{abstract}
Web 2.0 has brought a plethora of new tools (such as Twitter, Facebook, Instagram and others) and new functionalities, for instance, the ability to co-create web content. The question that is being asked is whether these applications and online tools can be used as an alternative to non-electronic tools for participation in spatial planning processes. The co-creation process that these tools enable is also participation. Participation in urban planning is an important part of space planning that we share with different users of planned space. The chapter shows how the theory of participation can be associated with participatory methods that are used in spatial planning. And how to use them when choosing and creating electronic Web 2.0 tools of. However, to make the use of electronic tools easier for non-professionals from the field of Information and Communication Technology (ICT), we have selected electronic tools designed for collaboration and participation to be integrated into a generative web framework. Various electronic tools are described in this chapter, with different ways of using in the processes of participation and co-creation. One tool that was developed for the needs of a certain European project from the field of integration of local initiatives in revitalizing urban public spaces of contemporary cities and could be used for other similar purposes will be described in more detail. An analysis of the tool used and the responses of those who used it will also be presented.
\end{abstract}

Keywords - Co-creation, public participation, web 2.0 


\section{INTRODUCTION}

Web 2.0 was first introduced in 2004 by O'Reilly (2005) and Dougherty, representatives of O'Reilly Media Publishing, as a concept that captured the emergence of new web pages. According to Berners-Lee (2006), Web 2.0 is not different from Web I.0, which in its principle put forward connections between users. Web 2.0 enables writing, which is basic for the participation of users and the development of social networks. Of the same opinion is Shuen (2008), saying that Web 2.0 is not about technology, but about web tools that enable people to work together, build and share information, experiences, photos and similar. Also, according to Alexander (2006), Web 2.0 is not a new discovery but is labelled as a mixture of similar technologies, useful on the Internet. Among them the »social« software application stands out as one of the main components. The term »social« means software that enables users and web developers or providers to make web pages more accessible to a wider range of users. Anderson (2007) similarly states that concepts like »cooperation«, »contribution « and »community« are present on the Internet daily and are part of a social network that is emerging »before the very eyes « of web users. This kind of network needs technologies that transform these concepts into web services and applications we use on the Web.

O'Reilly \& Battelle (2009) in their special report "Web Squared: Web 2.0 Five Years On" claimed that Web 2.0 is all about harnessing collective intelligence. Applications that enable collective intelligence must manage, understand and be able to process massive amounts of user-generated data in real time. Moreover, this is all the result of the mobile phone revolution from simple mobile phones to smartphones. Featuring integrated sensors (GPS, camera, microphone, heart rate and accelerometer) and many more sensors as add-ons (like sensors for measuring temperature, humidity etc.), smartphones can collect a vast amount of data in real time. The Web has moved from our desk computers to our smartphones, and the scale of participation has increased exponentially. Thus, in the case of massive external capture (Crowdsourcing), users or the public become human sensors that collect data, information, ideas and make suggestions (Chud \& Artigas, 2015).

With their applications and their sensors smartphones can help collect the knowledge of the crowds. Crowdsourcing is a relatively new concept, first mentioned by Howe (2006) in Wired magazine. Crowdsourcing is described as a type of participative online activity in which an individual, an institution, a non-profit organization, or company proposes to a group of individuals of varying knowledge, heterogeneity and number, via a flexible open call, the voluntary undertaking of a task (Estellés-Arolas \& González-Ladrón-de-Guevara, 20I2; Pedersen et al., 20I3). Crowdsourcing is a model capable of aggregating talent as well as reducing the costs and time to solve problems, and it is enabled only through the technology of the Web and its Web 2.0 tools (Brabham, 2008). 
Participation and co-creation in spatial planning processes are often restricted to techniques and tools that do not have any connection to the Internet. They are non-electronic tools, they are used at workshops, and public releases of plans prepared by planners or investors who want to find out public opinion on these matters. In the work "The Community Planning Handbook" Wates and Brook (2000) describe those techniques and tools (for example, photo survey, mapping, user groups etc.). The question that arises is whether the aforementioned techniques and tools can be implemented with the help of the Web 2.0 tools, thus enabling the public to co-create public open spaces or participate in urban planning also through the Internet successfully. What are these tools? If they are united in a single framework, are they therefore more accessible to the public for the purposes of co-creation and participation and will they reach more users than non-electronics tools and techniques? It is assumed that more creative public participation and co-creation could be ensured with new tools, emerging (daily) on the Internet. If the public were to be invited to help co-create open public spaces, they could bring suggestions, ideas and knowledge about those places in the community they live and work in via the internet. In many cases this could facilitate work for planners while at the same time drawing a less negative response from the public.

\section{THEORETICAL FRAMEWORK}

\section{Participation}

Participation in planning processes is a topic that has been discussed in many scientific fields. Pioneer in participation research, Arnstein (1969: 216) believes that "the idea of citizen participation is a little like eating spinach: no one is against it in principle because it is good for you". Moore \& Davis (1997: 5) are also very colourful in depicting the perception of people and their understanding of surroundings with an old Chinese proverb: "Tell me, and I will forget. Show me, and I may remember. Involve me, and I will understand". There are many theories of participation, and they address different types of participation (Arnstein, 1969; Waidemann \& Femers, 1993; Roche, 1997). Waidemann \& Femers (1993) presented a type of participation ladder based on the amount of information accessible to the public (Fig. I). This framing of the issue claims that the level of participation increases with the degree of public access to information. The more information is available to the public, the greater is their role in participation and their decision-making power.

From the perspective of information, technology-supported spatial planning, Waidemann \& Femers's participatory ladder is useful because participation levels are associated with information access. Therefore, it is the information in a specific process that is crucial for participants, and technology can make that information more readily available. Participatory models aim to enhance the power of individuals so they can influence decisions regarding their life. From the spatial planning point 


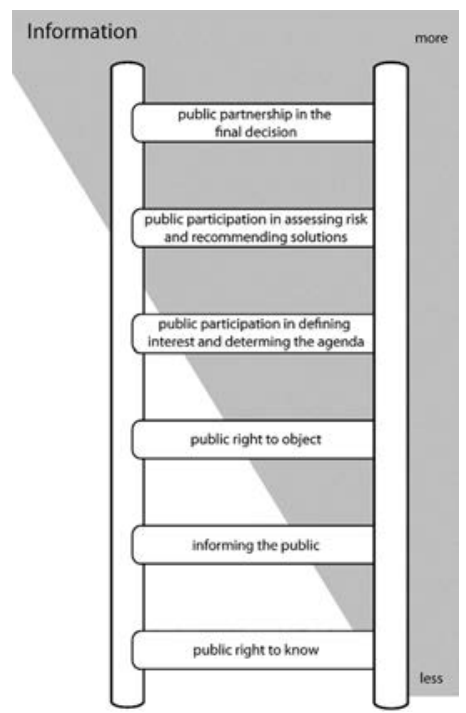

Fig. I: Public participation ladder, after Waidemann \& Femers (1993)

of view, Waidemann \& Femers's scale is the most appropriate. In this scale information and accessibility of information stand out as does, in the case of spatial planning, information about spatial interventions.

\section{Co-creation}

Co-creation involves people in more active participation dealing with public spaces. Among examples of such co-creation, two can be named: the so-called DIY urbanism and urban guerrilla movements. Examples include graffiti, parkour, yarn bombing and guerrilla knitting, seed bombing and guerrilla gardening, dîner en blanc, and Park(ing) Day (Foth, 2017). And co-creation has many multiple benefits, from generating useful information, creating a feeling of co-ownership, and building healthy relationships. Its benefits are increasingly apparent in many disciplines. The sciences have recognized the value of co-creation for co-ownership of information and social capital, particularly for locally contextualized work (Rock, McGuire \& Rogers, 2018). Because the challenges and risks are also well identified, workarounds will be evolving simultaneously. Is co-creation more than participation, is a question that Katrin Prager (2016) poses. She considers that both denote a process that involves active doing; both involve a collaborative process in which diverse stakeholders take part and both involve participants learning from each other. However, participation has many forms and not all forms of participation are appropriate for co-creation. The main difference, according to her, is that co-creation does not stop at actionable knowledge and it also requires practical outcomes. For example, co-creation is not only developing a joint action, but it also involves implementing it; in other words, it is not agreeing to redistribute funds, it is actually redistributing them. 


\section{Web 2.0 Tools and HTML5}

As we have ascertained, one of the most important novelties deriving from the concept of Web 2.0 is that users are adding data to the web and so co-designing web pages. By this, the administrators/owners of pages retrieve information about users, their knowledge and expertise that could be used for various purposes. Users can, therefore, participate or co-create, and owners gather knowledge from the crowd by crowdsourcing. Participation is enabled by web services and web applications. The basis for creating services and applications is a group of technologies by the name AJAX (Asynchronous Javascript and XML) (Anderson, 2007). Among basic services or tools that have emerged with Web 2.0 are blogs or weblogs, wiki pages, podcasts and video blogs, RSS protocol (Really Simple Syndication), beginning of tagging (tags), social bookmarks, social network services and web library (Alexander, 2006; Kolbitsch \& Maurer, 2006; Anderson, 2007).

In the following paragraphs, the basic Web 2.0 tools are described:

Blog or weblog is a diary on the Internet. It is based on the subjective presentation of contents and expresses the personal opinion of an author. It allows reader comments that the author responds to. An author can label entries with tags. This enables faster search through similar tags (Anderson, 2007). So, publishing blogs and commenting enables communication or expression of opinions by author and writers of comments (Benkler, 2006). A blog could be used to present an official stand of a planner or an expert opinion on the matter that the public present to planners or stakeholders.

Forum is a web 2.0 tool that enables the public to talk about everything in order to exchange opinions with experts, decision makers, stakeholders and planners. One such tool is an OPUS local online forum (Staffans, Rantanen \& Nummi, 2010). OPUS forums are a web concept where local knowledge, information and data are collected, analysed and transmitted. Forums are a learning platform where knowledge about the use of local land and development projects are interconnected. Forums offer a platform for partner projects and cooperation to local service providers, investors and planners.

Chat or Online chat refers to communication over the Internet that offers a real-time transmission of text messages among users of chatrooms. Such text messages are generally short in order to enable other participants to respond quickly. Participating in a chat provides a feeling similar to that of a spoken conversation (as in a meeting), which distinguishes chatting from other online communication such as Internet forums and email. The conversation can be recorded and later used as a meeting record (Carver, 2003).

Wiki is a web page prepared by one user that allows other users to add content, like the co-creation of a dictionary. The concept of wiki pages is that they enable fast and simple editing of web content by a collaboration of users, which means 
that a user inserts a text, another one edits it and yet another one complements it, etc. (Ebersbach \& Glaser, 2004). Wiki pages could be useful to clarify certain terminology in urban planning and to explain processes. But there are also numerous questions about co-creation, e.g. what happens if an author of the original text does not agree with further corrections and how to protect intellectual property (Lamb, 2004), and not least, the question of the open nature of wiki pages.

Tags are keywords, pinned to information, for instance to a photo, video, file or news. They contribute to a better description of information and an easier search through the web. Any user can pin tags to information and create his own list of tags or share them with other users (Högg et al., 2006; Maness, 2006; Anderson, 2007). Tags are useful to check on public opinion. For example, a solution for a new open place or the redesign of the park or town square could be published on the web and enabling users to express their opinion by pinning tags (like, 'don't like' and similar).

A podcast is similar to an audio diary, usually for recording lectures, interviews, radio shows, etc. On the other hand, videoblog is a recording of a similar kind, but for videos. Sound podcasts and video podcasts are very appropriate for presenting educational themes (Rogers, 2005; Anderson, 2007).

RSS protocol is intended to automatically disseminate data from web pages to users. This means that a user of RSS protocol does not have to search over and over again through web pages for information; rather he uses the installed RSS reader on the computer and subscription to a web page he wishes to receive information from (Anderson, 2007). The advantages of RSS protocol are that it automatically receives contents from RSS provider and thus allows users to concentrate on reading without wasting time searching web pages for new contents. It is also useful to report on events and procedures.

Web Geographic information system (WebGIS) is a group of tools that enables the presentation of spatial plans together with various specific maps (satellite images, plans of town infrastructure, three-dimensional objects, etc.). With Web 2.0 tools, GIS systems have allowed users to collaborate by drawing and adding spatial data to already prepared maps.

Web-based survey is a research tool that enables researchers to collect data from a variety of research fields, including public open spaces, urban planning, mobility and nature-based solutions, among others. Web-based surveys are faster and simpler than paper or e-mail surveys, the data collection period can be shortened as all the data is collected and processed almost at the same time. Questions of the web survey can have a pop-up help on the matter, and they can show errors to the respondent. The web-based survey is a consulting technique that provides means for planners/developers to gain feedback on proposals 
in ways that do not necessarily require citizens to attend a conventional public meeting (Jones, Layard, Speed \& Lorne, 20I5).

Social network service (SNS) or online social network is an online platform which enables people to build social networks or cultivate social relations with other people who share similar interests or activities. The networking functions of SNS serve to foster social relationships amongst users within the online platform. They provide functionality for building and maintaining the social network graph. The data functions are responsible for the management of user-provided content and communications amongst the users. Their variety contributes to the enhancement of users' interaction and makes the platform more attractive (Paul, Buchegger \& Strufe, 20I I).

In 20I4, World Wide Consortium (W3C) agreed on a new standard for HyperText Markup Language (HTML), called HTML5. Together with HTML5, they also introduced two other renewed standards: one for styles of web pages - Cascading Style Sheet 3 (CSS3) and one for Document Object Model (DOM). With those three technologies and Javascript, designers and programmers can now make mobile applications that can work on any web browser that supports HTML5 and on any device (desktop computer, notebook, tablets and smartphones). The most innovative features of HTML5 lie in its multimedia elements. Programmers can now use those elements (audio, camera, geolocation, local storage etc.) with ease and they can simply capture, for instance pictures from smartphone or conversation from a tablet or even geolocation from a desktop computer (Gutiérrez, 2017). With those new HTML5 elements, programmers are able to produce a completely new variety of apps for crowdsourcing, participation or co-creation that can be used on any platform (MS Windows, Linux or IOS) and on any device (desktop computer, notebook, tablets and smartphones).

\section{METHOD}

The research combines the theoretical study of participation and participation tools for community planning, co-creation, crowdsourcing and Web 2.0 tools from the literature to form a scientific base for practical work. Münster et al. (2017) stated that many offline participatory tools can be assisted by digital tools and that digital tools can be used instead of offline tools. The ideas of converting non-electronic participation tools to electronic ones was verified by using a working participatory system and making a working prototype tool to be used in a case study. Prototyping is a way to test a program or a tool. It is a real thing, not a design idea or concept. Moreover, as a real product, it can be tested, altered, and tested again until it meets the original idea or design (Warfel, 2009). The working system that has been used was the generative framework of various Web 2.0 tools that can be selected for various participation scenarios or co-creation tasks. The system has also various software components to enable programmers to make additional tools. So, methodology can, therefore, be regarded as action-research. 
The first step in the action-research was the definition of the necessary tool for carrying out the task. The next step was to investigate which software components make it possible to create a tool in the framework of the generative platform. The last step was to make a prototype tool and test it on a task within a certain EU project. This way, information was gathered on the kind of data that could be collected with the tool, how difficult it would be to make such tool, its usefulness, manufacturing errors and the extent of possible participation when used in a real environment scenario. And finally, information could be gathered on the public's responses to the tool.

\section{ANALYSIS AND DISCUSSION}

Spatial planning is a procedure that requires the inclusion of the public; acquiring initiatives and proposals that trigger the procedure of preparation or corrections to master plans and also forming standpoints in the matters of proposed solutions in public displays. Increasingly important is the use of the "wisdom of crowds" in planning - the knowledge and the sensibility the public has about their local community, where they live and in which they play an active role. On the other hand, planners in general see participation as an obstacle, not realizing the benefits to be had by using this knowledge. In most cases, one of the main problems is that harvesting public knowledge is a time-consuming and expensive process. Workshops have to be organized, questionnaires and other means of acquiring data prepared. Planners prefer to work and decide on the basis of expert studies that communities have had made prior to master plans. However, even this is not a rule. Crowdsourcing or mass outsourcing is one of the correct models to be used in urban planning participation because it combines the synergy, knowledge, views, and experiences of the crowd involved. At the same time, in the right circumstances the crowd is smarter than its smartest individual (Anttiroiko, 20I5). In the aforementioned work "The Community Planning Handbook" (Wates \& Brook, 2000) there are many useful methods that use various tools to gather the knowledge of the crowds, but they are all non-electronic. The book proposes that those tools can be used by planners or local community to organize workshops, field trips, simulations, design fests, etc. Award schemes, briefing workshops, choice catalogues, design games, (see Fig. 2) are some of the tools that can be transformed into Web 2.0 tools. Some of them (different versions of blogs, forums and chats programs, various types of Web GIS programs, many different ways to create web surveys etc.) can already be found on the Internet in abundance, and they can be used instantly on web pages, while other more complex ones are not immediately available and have to be designed. With those Web 2.0 tools the knowledge of the crowds can be captured as well as with the above-mentioned non-electronic tools. 


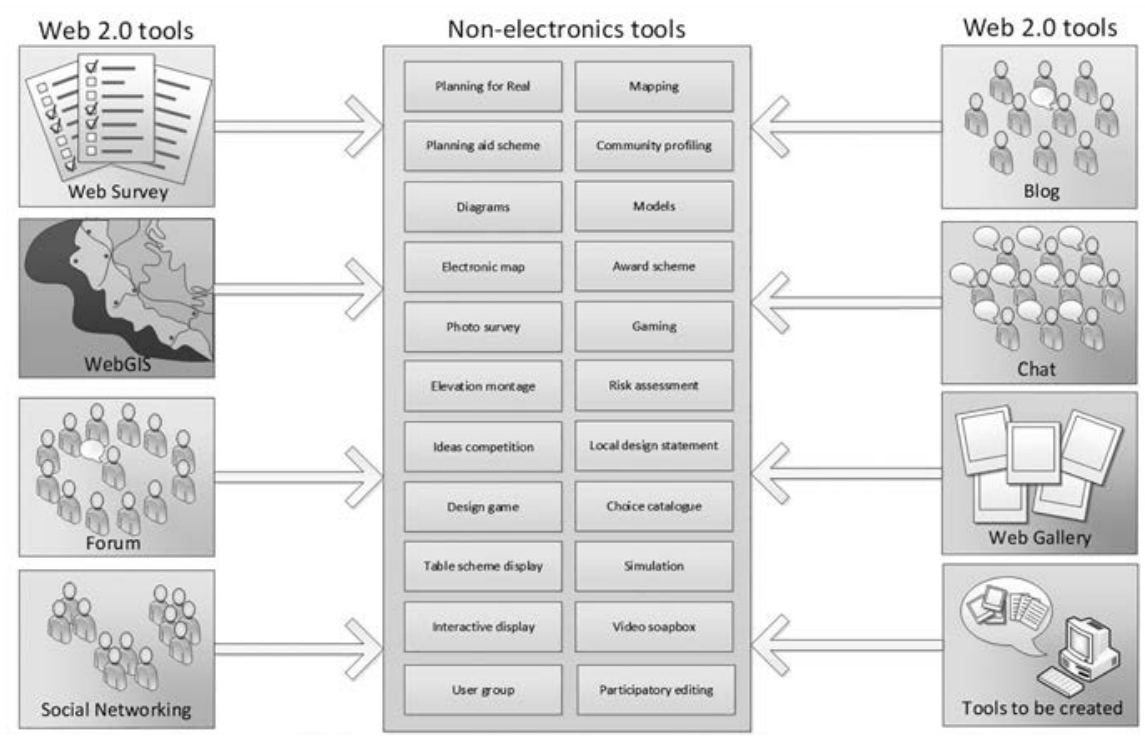

Fig. 2: Non-electronic tools can be converted into Web 2.0 tools. Source: Author.

According to a study on Internet usage in the EU in $2018,87 \%$ of households have access to the Internet, with Internet daily usage frequency of $72 \%$ of individuals. Of all the EU individuals, $67 \%$ have used a mobile phone to connect to the Internet. In $2018,56 \%$ of all EU individuals participated in social networks (creating a user profile, posting messages or other contributions to Facebook, Twitter, etc.) at some time (Eurostat, 2019). This indicates that more than half of the active population that could participate in procedures of spatial planning has access to the Internet, knows and uses some form of social networking tools and accordingly has greater ability for active participation. By using social networks in their current form, individuals are better qualified to access Web 2.0 tools. This was one of the bases for creating a generative platform for public participation.

Examining classical methods for harvesting public knowledge and learning about local communities has shown that certain methods can be simply translated to an electronic form and simultaneously used in the generative framework for public participation. In the same way that web GIS can be used as a substitute for a charting method or as an electronic map, that elements of social networks can be used as user groups, and that forums can be used as interactive displays or table scheme displays, photo galleries can be used instead of photo analyses or elevation photomontages. If urban planners want to use the bottom-up approach and use the knowledge of the masses, they should capture it by using Web 2.0 tools. Web 2.0 tools have the ability to support the collective knowledge of the masses due to their democratic, decentralized nature and the diverse functions they enable (Anttiroiko, 20I5). 
The generative framework of Web 2.0 tools for public participation was developed as a research project at The Urban Planning Institute of the Republic of Slovenia (UIRS). After initial tests, it was deployed on the UIRS infrastructure. To operate such framework, the UIRS has a specialized staff of professionals and experts in the fields of spatial planning and ICT, which can provide all the necessary infrastructure and knowledge for the support and further development of the framework, as well as all the necessary expert knowledge on spatial planning to provide the framework with a repository of expertise. The repository helps users learn about basic terms regarding urbanism, as well as processes and procedures regarding spatial planning. The framework is used in various projects, one of which being Human Cities (EU program Creative Europe). One of the tasks in the project was to give the public an opportunity to share photographs of their neighbourhood online (Nikšič, Goršič \& Tominc, 2018). A kind of gallery of pictures had been created, but it was necessary to complement the pictures with photographers' comments regarding them. In other words, coupling a gallery with a survey. A photographer was asked to answer a few questions (what the picture represents, what the photographer wanted to say to the public about the motive, etc.). So, each photography was fitted with attributes that had been gathered via an online form, guiding contributors through relevant questions about their contribution (theme, photography atmosphere, other information about the neighbourhood). All uploaded photographs had been put in a web gallery of the project and were later evaluated by a jury, awarding best contributions as some sort of stimulus for participatory.

For that task, the experts from the UIRS had created a new project package and interface within the generative framework and executed the programming. Within that project, they made a sub-portal with gallery tools, an additional tool in the form of an online questionnaire (Fig. 3) and another one for picture evaluation (Fig. 4). The tools were made using HTML5 language, Bootstrap 3 framework and Razor script. All those programming tools have become an integral part of the framework and can be used for further programming new tools. The sub-portal was set up in a few hours, new tools added in a week. The questionnaire within the tools enabled the public to take pictures with a mobile device or tablet or, if they have pictures on the notebook or desktop computer, to simply upload them from that device. The tool is programmed to add spatial coordinates automatically if the device was able to provide them. Conversely, the user was asked to enter the street name and neighbourhood which was then translated by Google Map services into spatial coordinates. Because users contribute pictures in five different sections, the jury tool was also divided into five categories. Each jury member ranked the top three pictures with 5, 4, or 3, 5 being the best. The tool then added all the scores and in the end showed the winning pictures in all five categories.

During the two-month phase of the photo story contest, 172 pictures were collected from 79 users. There were 52 different neighbourhoods presenting 


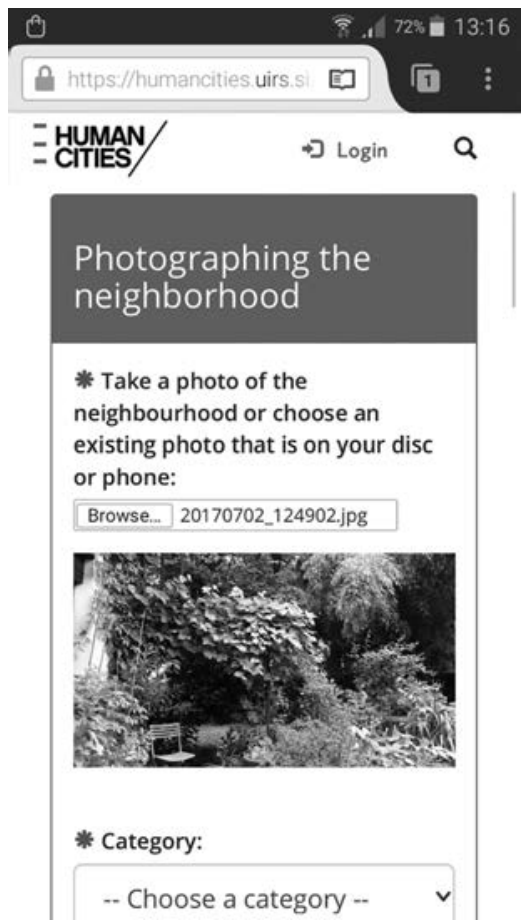

Fig. 3: Mobile device screen capture from the online questionnaire for the EU project by UIRS.

Source: Author.

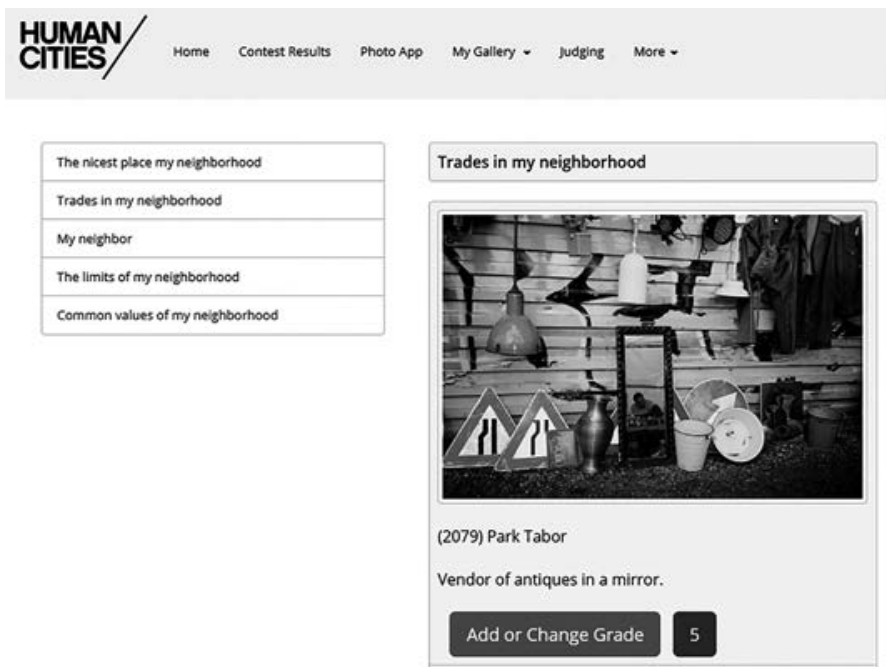

Fig. 4: Tool for jury/competition task. Source: Author.

pictures in five different descriptive categories. During the time of the photo story contest 675 users visited the web page, making I,I 83 sessions, and 2,859-page views with a $62 \%$ bounce rate. In I, 183 sessions, $74 \%$ of users accessed the web page from desktop computers, $22 \%$ from mobile devices and $4 \%$ from tablets. Out of $79 \%$ users, 
$5 \%$ were younger than 14 years of age, $27 \%$ were between 15 and $24,33 \%$ between 25 and 34, 19\% between 35 and 44, 7\% between 45 and 54, 7\% between 55 and 64 and $3 \%$ older than 65 . Participation by gender was divided into $32 \%$ males and $68 \%$ females. Among them were $41 \%$ were still in school, $43 \%$ were employed, $8 \%$ unemployed and $8 \%$ retired. There were no major problems with the use of the questionnaire tool exhibited. It was concluded from the statistic that people still prefer to use desktop computers over smartphones and tablets. The majority of them was 15 to 44 years old. There were some problems with on-site registration, especially by elderly users $(65+)$. The registration consisted of an on-site registration form and confirmation of the registration by clicking the confirmation link in the return e-mail from the site. Some of those return e-mails had ended in the junk mail folder, and many elderly people were not aware of this or could not find it. But overall satisfaction with the questionnaire and the tools for the jury was good. The users were also asked to express their opinion on the portal. The prevalent answers were that with some minor modifications the tools are useful for many different tasks involving capturing and evaluating pictures for the competition.

\section{CONCLUSION}

The concepts were confirmed by building a prototype tool that had been proven so by the analysis. Because the tools' software environment is flexible, it is possible to adjust the tool according to various questions in the survey. The use of the generative framework and the tool had shown that it was reasonably easy to set up a participatory system by enhancing a planning project with social tools. The framework with its tools can assist a bottom-up civil initiative as well by creating a web-based platform that includes open public information. The advantages of this framework are mostly in terms of flexibility as well as the ease of developing, adding and upgrading those tools in the future. The framework's flexibility and extension potential are possible because the framework combines the theory of participation with the theories of information systems and social media tools. The framework and its tools are versatile enough to perform different tasks for collecting public opinion and knowledge about the public open spaces and co-creation of those spaces.

The power of the Web 2.0 tools lies, among other things, in the co-creation taking place among their users. Co-creation could be named participation with a concrete result. In the presented case, the photographs and the results of the completed survey became input data for the planners of the open space in the neighbourhood to make it more pleasant to its dwellers. Users of Web 2.0 tools can participate with their opinions in the problems of open space, by choosing the offered solutions, or by offering better solutions, etc. Insofar as their proposals and solutions are taken into account in the final implementation of the project, this means that they have co-created the open space (Daiberl, 2017; Parmentier \& Rolland, 2013; Nandi \& Mittal, 20I4). 
There are many questions that can be asked when preparing for participation and co-creation, such as: how to reach and involve public representatives, how to involve and activate the public to cooperate from the start of the project, how to present a project in an understandable way, and how to organize participation or co-creation for the sake of users. Some of those questions can be answered with various communications channels between planners and public. Communication can be either one-way (e.g. advertising, media, direct mailings) or two-way (workshops, boots, website, apps, social media etc.), physical or electronic (virtual). But only two-way communication can provide participation and co-creation; on the other hand, one-way communication can be used to convey information about the project to the public at the earliest stage of the project (Münster et al., 2017). By using the generative framework and its tools, users will leave digital trails in the framework. And those digital trails, which include suggestions and ideas of public open spaces, will lead to "big data" on people's perception of space. This will present a challenge for researchers who wish to study them. Still, grasping this will lead to a better understanding of public spaces and will help to shape the environment in which we live in.

\section{REFERENCES}

Alexander, B. (2006). Web 2.0: A new wave of innovation for teaching and learning? Educause Review, $4 \mathrm{I}(2)$ : 32-44.

Anderson, P. (2007). What is web 2.0? Ideas, technologies and implications for education. JISC Technology \& Standards Watch. Retrived from www.jisc.ac.uk.

Anttiroiko, A. (2015). Smart Planning: The potential of Web 2.0 for Enhancing Collective Intelligence in Urban Planning. Ed. Carlos Nunes Silva: Emerging Issues, Challenges, and Opportunities in Urban E-Planning, I-32. Engineering Science Reference - IGI GLobal. Hershey, USA.

Arnstein, S. R. (1969). A ladder of citizen participation. Journal of The American Institute of Planners, 35, 216-224. DOI: 10.1080/01944366908977225

Benkler, Y. (2006). The wealth of networks: How social production transforms markets and freedom. New Haven, Yale University Press.

Berners-Lee, T. (2006). Tim Berners-Lee. Ed. Scott Laningham: DeveloperWorks Interviews. Podcast. Retrieved from https://www.ibm.com/developerworks/podcast/dwi/cm-int082206txt.html.

Brabham, D. C. (2008). Crowdsourcing as a Model for Problem Solving: An Introduction and Cases. Convergence: The International Journal of Research into New Media Technologies I4 (I): 75-90. SAGE Journals. New York, USA.

Carver, S. (2003). The Future of Participatory Approaches Using Geographic Information: developing a research agenda for 2 Ist Century. Journal of the Urban and Regional Information System Association, 15, Special Issue, 6I-7I. Illinois, USA.

Chud, S. A. \& Artigas, F. (2015). Participatory Environmental Planning Platform. Ed. Carlos Nunes Silva: Emerging Issues, Challenges, and Opportunities in Urban E-Planning, 46-68. Engineering Science Reference - IGI GLobal. Hershey, USA.

Daiberl, C. (2016). Online-Offline Co-Creation. Open Service Lab Notes. Chair of Information Systems I - Innovation \& Value Creation. Nuremberg, Germany. 
Ebersbach, A. \& Glaser, M. (2004). Towards emancipatory use of a medium: The Wiki. International Journal of Information Ethics, I I (2), I-9.

Estellés-Arolas, E. \& González-Ladrón-de-Guevara, F. (2012). Towards an integrated crowdsourcing definition. Journal of Information Science 38 (2): I89-200. SAGE Journals. New York, USA.

Eurostat (2019). The statistical portal. Retrieved from https://ec.europa.eu/eurostat

Foth, M. (2017). Participation, Co-Creation, and Public Space. The Journal of Public Space 2 (4): 2 I- 36. City Space Architecture. Bologna, Italy.

Gutiérrez, R. T. (2017). Taking a Glance at the History of HTML5. Conference: European Academy of Standardization Conference. EURAS 2017. Berlin, Germany.

Howe, J., (2006). The Rise of Crowdsourcing. Wired Magazine I4 (6). Condé Nast, San Francisco, USA.

Högg, R., Meckel, M., StanoevskaSlabeva, K. \& Martignoni R. (2006). Overview of business models for Web 2.0 communities. The article was presented at the conference GeNeMe 2006, 28-29. September. Dresden, Germany.

Jones, P., Layard, A., Speed, C., \& Lorne, C. (20I5). MapLocal: Use of Smartphones for Crowdsourced Planning. Planning Practice \& Research 30 (3): 322-336. Routledge - Taylor \& Francis Group. Abingdon, UK.

Kolbitsch, J. \& Maurer, H. (2006). The transformation of the web: How emerging communities shape the information we consume. Journal of Universal Computer Science, 12(2): 187-213.

Lamb, B. (2004). Wide open spaces: Wikis, ready or not. EDUCAUSE Review, 39(5): 36-48.

Maness, J. M. (2006). Library 2.0 Theory: Web 2.0 and Its Implications for Libraries. Webology, 3 (2). Retrieved from http://www.webology.org/2006/v3n2/a25.html

Moore, C. N. \& Davis, R. (1997). Participation tools for better land-use planning. Sacramento, USA, Centre for livable communities.

Münster, S., Georgi, C., Heijne, K., Klamert, K., Noenning, J. R., Pump, M. et al. (2017). How to involve inhabitants in urban design planning by using digital tools? An overview on a state of the art, key challenges and promising approaches. Procedia Computer Science, 2391-2405. Elsevier. Amsterdam, Netherlands.

Nandi, V. T., \& Mittal, H. K. (20I4). Co creation and collaboration: branding tools. International Journal of Knowledge and Research in Management \& E-Commerce 4 (3): II-I8.

Nikšič, M., Goršič, N. \& Tominc, B. (2018). Tools for participatory provision of urban public spaces: Human Cities experience. Human cities: Challenging the City scale 2014-20I8 / Investigation, Emilie Chabert (Eds.), 9-25. Cité du design Saint-Étienne, France.

O'Reilly, T. (2005). What Is Web 2.0: Design Patterns and Business Models for the Next Generation of Software. O'Reilly. USA. Retrieved from http://www.oreillynet.com/pub/a/oreilly/tim/news/2005/09/30/ whatis-web-20.html

O’Reilly, T. \& Battelle, J. (2009). Web Squared: Web 2.0 Five Years On. Web 2.0 summit. O'Reilly. USA. Retrieved from https://conferences.oreilly.com/web2summit/web2009/public/schedule/detail/I 0194

Paul, T., Buchegger, S., \& Strufe T. (20II). Decentralized Social Networking Services. Trustworthy Internet, Blefari-Melazzi, Nicola, Bianchi, Giuseppe, Salgarelli, Luca (Eds.), 187-199. Springer International Publishing. Netherlands.

Parmentier, G., \& Rolland, S. (20I3). The benefit of social media: Bulletin board focus groups as a tool for co-creation. International Journal of Market Research 55 (6), 809-827. SAGE Journals. New York, USA.

Pedersen, J., Kocsis, D., Tripathi, A., Tarrell, A., Weerakoon, A., Tahmasbi, N. \& De Vreede, G. J. (20I3). Conceptual foundations of crowdsourcing: a review of IS research. In System Sciences (HICSS), 2013 46 $6^{\text {th }}$ Hawaii International Conference on (PP. 579-588). IEEE. 
Prager, K. (2016). Is co-creation more than participation? [Blog post]. Retrieved from https://i2insights. org/2016/07/28/co-creation-or-participation/.

Rocha, E. (1997). A ladder of empowerment. Journal of Planning Education and Research, I7(I), 3I-44. DOI: $10.1177 / 0739456 \times 9701700104$

Rock, J., McGuire, M. \& Rogers, A. (2018). Multidisciplinary Perspectives on Co-creation. Science Communication 40 (4): 54I-552. SAGE Journals. New York, USA.

Rogers, G. P. (2005). Podcasting and its role in semantic social networks, the Web 2.0, and the semantic web. Special Issue on Semantic Web and Social Networks, AIS SIGSEMIS Bulletin, 2(3/4): 57-6I.

Staffans, A., Rantanen, H. \& Nummi, P. (20I0). Online Environments Shake up Urban Planning - Developing Local Internet Forums. Digital tools in participatory planning. Aalto, Finska, Aalto University.

Shuen, A. (2008). Web 2.0: A strategy guide. Sebastopol, O'Reilly Media.

Waidemann, I. \& Femers, S. (1993). Public participation in waste management decision making: Analysis and management of conflicts. Journal of Hazardous Materials, 33(3), 355-368. DOI: 10.1016/0304-3894 (93)85085-S

Wates, N. \& Brook, J. (2000). The Community Planning Handbook. London, Velika Britanija, Earthscan Publications Ltd.

Warfel, T. Z. (2009). Prototyping. Rosenfeld Media, New York, USA. 